Sonia E. Alvarez

University of Massachusetts, Amherst

\title{
Construindo uma política feminista translocal da tradução'
}

\begin{abstract}
Resumo: Nosso projeto coletivo Translocalities/Translocalidades: Feminist Politics of Translation in the Latin/a Américas (Políticas Feministas de Tradução na América Latina) explora como discursos e práticas feministas viajam por uma variedade de lugares e direções e acabam se tornando paradigmas interpretativos para a leitura/escrita de questões de classe, gênero, sexualidade, migração, saúde, cidadania, política e circulação de identidades e textos. Sustentamos que a tradução é política e teoricamente indispensável para forjar epistemologias e alianças políiticas feministas, antirracistas e pós-coloniais/pós-ocidentais, pois as Américas Latinas - enquanto formação cultural transfronteiriça e não territorialmente delimitada - devem ser entendidas como translocais em dois sentidos. O primeiro sentido que usamos - o de translocalidade - parte de movimentos além das concepções da "política da localização" empregadas pelo feminismo terceiro-mundista estadunidense. Mais do que "migrar" e "se assimilar", muitas pessoas nas Américas Latinas cada vez mais se movem de um lado para outro entre localidades, entre lugares historicamente situados e culturalmente específicos, ainda que porosos, atravessando múltiplas fronteiras, e não apenas entre nações (como deixa a entender o termo "migração transnacional", por exemplo). Empregamos a expressão translocal, então, em um segundo sentido, que chamamos de translocalidades, precisamente para capturar esses cruzamentos e movimentos multidirecionais. Palavras-chave: tradução; feminismos transnacionais; raça/racismo; feminismos latinoamericanos; latinas.
\end{abstract}

Copyright (c) 2009 by Revista Estudos Feministas.

1 Extraído de "Introductions to Feminist Politics of Translation I," In Translocalities/Trans/ocalidades: Feminist Politics of Translation in the Latin/a Américas, organizado por Sonia E. Alvarez, Claudia de Lima Costa, Verónica Feliu, Rebecca Hester, Norma Klahn e Millie Thayer, com Cruz C. Bueno. Contribuidor@s incluem autoras da Argentina, Brasil, Bolívia, Chile e México, além de Latin@s de descendência cubana, portoriquenha, mexicana, chilena, peruana e dominicana, e outras
Nosso projeto coletivo Trans/ocalities/Trans/ocalidades: Feminist Politics of Translation in the Latin/a Américas (Políticas Feministas de Tradução na América Latina) ${ }^{2}$ explora como discursos e práticas feministas viajam por uma variedade de lugares e direções e acabam se tornando paradigmas interpretativos para a leitura/escrita de questões de classe, gênero, sexualidade, migração, saúde, cidadania, política e circulação de identidades e textos. Empregamos a noção de tradução de forma figurada, para enfatizar como essas viagens estão mergulhadas em questões mais amplas de globalização e envolvem trocas entre várias localidades, especialmente entre mulheres na América Latina e latinas nos Estados Unidos. Constituímos uma política de tradução ao despudoradamente traficarmos teorias e práticas 
"mulheres de cor" e aliadas dos Estados Unidos. Estou particularmente endividada neste ensaio e no projeto como um todo com Claudia de Lima Costa, por ter me convidado a viajar com ela nas questões de tradução feminista (e muitas outras que têm sido tanto profissional quanto pessoalmente enriquecedoras). Sem nossos próprios trans/deslocamentos e sem seu impressionante e amplo comando das teorias feministas de tradução, transnacionalismo e tradução cultural em geral, este projeto coletivo e este ensaio em particular não teriam sido possíveis. Agradeço também a tod@s@s Transloc@s que conosco empreenderam esta jornada ao longo desses muitos anos. ${ }_{2}$ Deve-se atentar para o uso do termo "Latin/a América" pela autora. A expressão é composta de um jogo de palavras que se desdobra em Latin America = América Latina, Latina $=$ termo usado para se referir a pessoas de origem latino-ameicana residentes no norte do continente e no uso da palavra América, em sua forma em português e espanhol, ao invés de sua forma em inglês, America. Diante da dificuldade de expressar todas essas nuances, optou-se por traduzir o termo como América Latina, salientando desde o início seu caráter polissêmico. (N.T.)

${ }^{3}$ Para um panorama do debate feminista sobre a política da localização, ver Kathy DAVIS, 2007, p. 7-11.

${ }^{4}$ LAÓ-MONTES, 2007, p. 122.

${ }^{5}$ LAÓ-MONTES, 2007. Ver também Agustín LAÓ-MONTES e Mirangela BUGGS, no prelo.

${ }^{\circ}$ CARRILLO, no prelo. feministas, cruzando fronteiras geopolíticas, disciplinares e de outras naturezas, trazendo insights dos feminismos de latinas, de mulheres de cor e do feminismo pós-colonial do norte das Américas para as nossas análises de teorias, práticas, culturas e políiticas do Sul, e vice-versa.

A tradução é política e teoricamente indispensável para forjar epistemologias e alianças políticas feministas, antirracistas e pós-coloniais/pós-ocidentais, pois as Américas Latinas - enquanto formação cultural transfronteiriça e não territorialmente delimitada - devem ser entendidas como translocais em dois sentidos. O primeiro sentido que usamos - o de translocalidade - parte de movimentos além das concepções da "política da localização" empregadas pelo feminismo terceiro-mundista estadunidense. Já que uma política feminista da localização envolve "uma temporalidade de luta, e não uma posição fixa", como argumenta Cláudia de Lima Costa (no prelo), devemos estar atentas às relações sociais e de poder que "produzem conhecimentos locais e situados". ${ }^{3}$ Contudo, como Agustín Laó-Montes sugere, latinas/os, e afro-latinas/os em particular, são mais entendidas/os como "sujeitos translocais". Nessa perspectiva, a política da localização, como é desenvolvida pelos feminismos das mulheres de cor nos Estados Unidos, "relaciona as 'múltiplas mediações' (gênero, classe, raça etc.) que constituem o self aos diversos modos de dominação (capitalismo, patriarcado, racismo, imperialismo) e às distintas, ainda que conectadas, lutas e movimentos sociais". ${ }^{4} \mathrm{~A}$ noção de translocal dá um passo à frente, vinculando "geografias de poder em várias escalas (local, nacional, regional, global) a posições de sujeitos (gênero/sexual, étnico-racial, classe etc.) que constituem o self". ${ }^{5}$ Nós ampliamos esse conceito de translocal para englobar não apenas latinas/ os estadunidenses, mas de todas as Américas Latinas.

Uma política hemisférica do translocal deve dar atenção à heterogeneidade das Latinidades dentro dos Estados Unidos e entre povos latino-americanos e caribenhos, bem como às várias posicionalidades que modelam as vidas latino-americanas através das múltiplas fronteiras. Muitos tipos de latino-americanidades - afro, queer, indígena, feminista - são construídos através de processos translocais. A Latinidad no sul, norte e Caribe já é liminarmente constituída de interseções dos fluxos intensificados transfronteiriços, transculturais e translocais que caracterizam a transmigração contemporânea pelo Hemisfério - de La Paz a Buenos Aires a Chicago e de volta. Teresa Carrillo ${ }^{6}$ esclarece que muitos desses cruzamentos são emocional, material e fisicamente custosos, e cada vez mais perigosos. No entanto, o cruzamento de fronteiras também sempre "reposiciona" e transforma subjetividades e visões do mundo. 
${ }^{7}$ MILLÁN, no prelo.

${ }^{8}$ Edward SAID, 1983, citado por Claudia de LIMA COSTA, no prelo.

${ }^{9}$ Sobre a política feminista do lugar, ver Wendy HARCOURT e Arturo ESCOBAR, 2005.

${ }^{10}$ MAIA, no prelo.
Mais do que "migrar" e "se assimilar", muitas pessoas nas Américas Latinas cada vez mais se movem de um lado para outro entre localidades, entre lugares historicamente situados e culturalmente específicos, ainda que porosos, atravessando múltiplas fronteiras, e não apenas entre nações (como deixa a entender o termo "migração transnacional", por exemplo). Empregamos a expressão translocal, então, em um segundo sentido, que chamamos de translocalidades, precisamente para capturar esses cruzamentos e movimentos multidirecionais.

Muitas feministas e outras teóricas críticas transitam, hodiernamente, por uma série de circuitos íntimos, familiares, libidinais, culturais, financeiros, políticos e trabalhistas, dentro de e atravessando diferentes locais das Américas Latinas e além. Nosso feminismo, como sugere Margara, ${ }^{7}$ é uma "prática multilocalizada". Assim como as "teorias viajantes" 8 e os transmigrantes de hoje, nossos próprios cruzamentos - teóricos, políticos, pessoais e íntimos - são pesadamente patrulhados e frequentemente obstruídos por diversos tipos de vigilantes (patriarcais, disciplinares, institucionais, capitalistas/neoliberais, geopolíticos, sexuais e por aí vai), como bem nos lembra Lima Costa.

Nossos múltiplos "locais" ou posições de sujeito mudam, de forma crucial para a política da tradução, de acordo com nossos movimentos e passagens por "localidades" espaço-temporais. Nossas subjetividades são, ao mesmo tempo, baseadas no lugar e des-locadas ou malcolocadas. ${ }^{9}$ Se eu sou uma cubano-americana "étnica" no sul da Flórida e uma latina racializada na Nova Inglaterra, no momento em que pouso em São Paulo eu me "torno branca". Mas incorporo essa "branquitude" provisória de maneira desconfortável, pois tenho consciência das injúrias infligidas pelo racismo tanto no norte como no sul das Américas. Apesar de menos flexível para os corpos mais negros devido ao "fato da negritude", a raça pode ser um significante móvel através das fronteiras. Como deixa claro a antropóloga brasileira Suzana Maia, ${ }^{10}$ "a raça não é um marcador fixo de identidade, mas varia de acordo com os lugares em que se habita".

Porque nosso trânsito através de múltiplas bordas perturba o senso comum em muitas das localidades nas quais nos movimentamos, de forma que às vezes nos faz parecer que perdemos o juízo, desde cedo adotamos o apelido de "Translocas" para nosso grupo de pesquisa transdisciplinar e transfronteiriço de feministas latinas e latino-americanas. Propomos agora Translocas como um projeto político e uma episteme para apreender e negociar as Américas globalizadas, um projeto que pode ser abraçado amplamente no Hemisfério ou mesmo além dele. 
11 Inderpal GREWAL e Caren KAPLAN, 1994, p. 7.

12 CÉSPEDES, 2007, p. 107; Maria LUGONES, 1990.

${ }^{13}$ SANDOVAL, 1991, 2000.

${ }^{14}$ ASHER, no prelo.

${ }^{15}$ HALL, 2003, p. 27, citado por Simone SCHMIDT, no prelo.

16 LIMA COSTA, 2000, p. 728.
De fato, com a intensificação da transmigração, um crescente número de latinas/os e latino-americanas/os incorporam registros, posições e epistemes cambiantes devido a nosso movimento intermitente dentro e cruzando as diversas localidades no norte e sul das Américas. Um crescente número de pessoas está, na verdade, tornando-se Transloc@s. Expandimo-nos exponencialmente.

Translocas nas Américas e em outros lugares globalizados desafiam o paradigma do "nós e eles", que deriva dos modos de descrição e representação modernista/ [colonial], "pois somos, simultânea e intermitentemente, nós e o outro"." Karina Céspedes aponta que muitas pessoas nas Américas Latinas são viajantes mundiais "por necessidade e sobrevivência". ${ }^{12}$ As viagens e traduções das Translocas são também conduzidas por afetos, paixões, solidariedade, conexões interpessoais e políticas. Além disso, viajamos por múltiplos mundos dentro de nós. Mais do que a "dupla consciência" de DuBois, nossa translocalidade permite uma consciência múltipla, interseccional, multilocal, uma versão "translocada" do que a Chela Sandoval chama de consciência diferencial ou oposicional. ${ }^{13}$ Muitas de nós tornaram-se "agentes duplas," termo usado por Kiran Asher para descrever sua própria "translocalidade" como feminista sul-asiática que pesquisa e trabalha com mulheres afrocolombianas. ${ }^{14}$

Simone Schmidt defende que o deslocamento é algo muito familiar aos sujeitos da modernidade tardia, e o sentimento de "des-locamento", ou, nesse caso, de trans-localidade, comumente nos deixa, como sugerido por Stuart Hall, com a sensação de "não estarmos em casa", em lugar algum. ${ }^{15}$ Ela argumenta que talvez seja apropriado "pensar que não é possível voltar para casa, pois o lar já não existe, pois o caminho para longe de casa é uma estrada sem volta". Talvez como Anzaldúa, que, Schmidt lembra, dizia carregar sua casa nas costas como uma tartaruga, nós Translocas também carreguemos nossas localidades em nossas costas.

Nossos deslocamentos por vários "aquis" e "alis", nossas "viagens por diferentes contextos de produção e recepção de conhecimento", como sugerido por Lima Costa, dão às Translocas "certo tipo de bagagem analítica que pode alterar a percepção da subalternidade, privilégio, trabalho intelectual e feminismo". ${ }^{16}$ Assim como Ester Shapiro, muitas de nós "lutamos para aprender com nossas re-localizações... como forasteiras culturais em organizações feministas etnocêntricas estadunidenses; como mulheres em organizações machistas da comunidade latina; como mulheres do 'terceiro mundo' cujo espanhol é primitivo e caribenho demais para sensibilidades europeias; e como 'gringas latinas' cujo 
17 SHAPIRO, no prelo.

${ }^{18}$ ESPINAL, no prelo. espanglês nos marca como não suficientemente educadas na língua, cultura e política da nossa nação de origem."17

Devido a nossos vários circuitos, viagens, deslocamentos e reposicionamentos, nós Translocas somos mais do que sujeitos da diáspora; somos necessariamente tradutoras. Para começar, temos que nos traduzir através dos diversos locais em que temos relações e compromissos. De fato, para aquelas de nós que moramos nos EUA, a tradução é um "jogo sem descanso", um "modo de vida, uma estratégia de sobrevivência no norte", como diz Isabel Espinal. ${ }^{18}$ Para muitas de nós que nasceram nos EUA ou que imigraram quando criança com pais que não falavam inglês, a "tradução começa praticamente na infância". Translocas expandem e transformam línguas e culturas, de forma que nem a "língua-materna" nem nossa(s) "outra(s)" língua(s) são "realmente estrangeiras" ou "nossas", Espinal explica. Assim como Donna Kaye Ruskin, cujo "The Bridge Poem" (O poema (da) ponte) abre This Bridge Called My Back: Writings by Radical Women of Color, nós "traduzimos mais do que / o raio das Nações Unidas". Ruskin reclama de estar cansada de traduzir: l've had enough

I'm sick of seeing and touching

Both sides of things

Sick of being the damn bridge for everybody

\section{Nobody}

Can talk to anybody

Without me

Right?

I explain my mother to my father my father to my little sister

My little sister to my brother my brother to the white feminists

The white feminists to the Black church folks the Black church folks

To the ex-hippies the ex-hippies to the Black separatists the

Black separatists to the artists the artists to the my friends' parents... Then I have to explain myself To everybody.
Cansei

Estou cansada de ver e tocar

Os dois lados das coisas

Cansada de ser o raio de uma ponte para todo mundo

\section{Ninguém \\ Consegue falar com o outroSem mim}

\section{Certo?}

Eu explico a minha mãe para o meu pai meu pai para minha irmã mais nova Minha irmã mais nova para o meu irmão meu irmão para as feministas brancas As feministas brancas para o povo negro da igreja o povo negro da igreja Para os ex-hippies os ex-hippies para os separatistas negros

Os separatistas negros para os artistas os artistas para os amigos de meus pais...

Aí eu tenho que me explicar

Para todo mundo.

Senti-me motivada a revisitar o poema de Ruskin no processo de escrever este ensaio e acabei fazendo uma "adaptação transloca personalizada": 
I sometimes grow weary of seeing and touching

Multiple sides of things

I explain the "Americanos" to the Cubans the Cubans to the Brazilians the (racist) Brazilians (and Cubans and other Latin Americans) to the US Women of Color feminists the US Women of Color feminists to Latino men the Latino men to the US white feminists the US white feminists to the Latin American Black feminists and to the Latin American white feminists who don't identify as white the Latin American white feminists to queer US Latinas the queer US Latinas to my mother's Cuban-American friends...

Then I try to explain myself To everybody.
Às vezes me canso de ver e tocar

Os múltiplos lados das coisas

Explico os "americanos" para os cubanos os cubanos para os brasileiros os (racistas) brasileiros (e cubanos e outros latino-americanos) para as mulheres de cor feministas dos EUA as mulheres de cor feministas dos EUA para os homens latinos os homens latinos para as feministas brancas as feministas brancas para as feministas negras latinoamericanas e para as feministas brancas latino-americanas que não se identificam como brancasas feministas brancas latinoamericanaspara as latinas queer dos EUA as latinas queer dos EUA para as amigas cubano-americanas de minha mãe...

Aí eu tento me explicar Para todo mundo.

\footnotetext{
19 MOHANTY, citado por THAYER, no prelo.

${ }^{20}$ LAÓ-MONTES, 2007, p. 132.
}

Espinal lamenta que esse tipo de tradução multidirecional "pode se tornar entediante e nós acabarmos hartos desse papel". No entanto, Translocas como Espinal e eu não podemos nos cansar de traduzir. Diante de um crescente aprisionamento de culturas e conhecimentos locais nos fluxos globais de capital e mercadoria, surge a necessidade de um engajamento, por parte das feministas, em diálogos e negociações produtivas através de múltiplas fronteiras geopolíticas e teóricas. Millie Thayer sugere que "as apostas na tradução feminista são grandes"; as próprias traduções, ela defende, são "objetos de luta" e a "tradução, ou a recusa de traduzir, são atos políticos estratégicos, quer envolvam o compartilhamento de conhecimento para forjar alianças ou a interrupção de um discurso dominante". Se os movimentos de mulheres na América Latina e em outros lugares do sul global partilham um "contexto comum de luta", como Thayer aponta, então "seus conflitos com as 'hegemonias espalhadas' representadas por Estados, indústria, capital global, fundamentalismos religiosos e relações de mercado criam poderosos, mesmo que parcialmente sobrepostos, interesses e identidades que tornam possível e ainda mais premente o projeto de tradução entre eles". ${ }^{19}$

Precisamos construir "epistemologias conectantes" 20 para confrontar as traduções errôneas ou malfeitas que estimularam a falta de compreensão e obstruíram alianças feministas, mesmo entre as mulheres que compartilham as mesmas línguas e culturas - como as latinas que residem nos EUA e as latino-americanas. Lima Costa argumenta que "nas interações entre os feminismos de latinas e de latino- 
${ }^{21}$ LIMA COSTA, no prelo.

${ }^{22}$ LAÓ-MONTES E BUGGS, no prelo.

${ }^{23}$ SHAPIRO, no prelo.

${ }^{24}$ ORTIZ, 1970, citado por Priscila RENTA, 2007.

${ }^{25}$ LIMA COSTA, no prelo.

${ }^{26}$ Ginetta CANDELARIO, 2007, relata os processos de racialização entre dominicanos em Nova York, Washington DC e Santo Domingo, afirmando que identidades e autopercepções raciais baseadas na nacionalidade podem ser bastante resistentes, mesmo quando sujeitos cruzam fronteiras.

${ }^{27}$ CARRILLO, no prelo.

${ }^{28}$ Jorge DUANY, 2008. americanas, a circulação de discursos e práticas encontra bloqueios enormes e pontos de controle de migração". Ela reconta a luta (e frustração) incessante de nosso grupo Translocas contra, de um lado, a "(im)possibilidade de tradução do conceito estadunidense de 'mulher de cor' quando levado a outras topografias" e, do outro, contra o apagamento de questões de sexualidade, raça e classe na produção de um "sujeito universal do feminismo latino-americano" - apagamento esse que é produto de operações que Millán chama de "feminismo hegemônico" no norte-dentro-do-sul das Américas. ${ }^{21}$

As Américas Latinas são compostas de diásporas múltiplas e multidirecionais, muitas vezes sobrepostas e interconectadas. Teóricas e ativistas latinas de cor, principalmente feministas antirracistas e defensoras dos direitos de indígenas e de afro-latinas, são particularmente bem "translocalizadas" para ajudar a construir pontes entre identidades e epistemologias pelas Américas. Laó-Montes e Buggs ${ }^{22}$ sustentam, por exemplo, que a diferença afro-latina pode ser um componente crucial em uma comunidade de alianças políticas e um elemento significativo nos campos de produção e crítica intelectual. E como sugerido por Shapiro, imigrantes latinas nos EUA também podem dar uma "importante contribuição para a tradução de ativismos feministas perpassando as divisões estadunidenses de raça, etnicidade, classe e status educacional, enquanto permanecem associadas aos feminismos globais do terceiro mundo através das conexões com seus países de origem". ${ }^{23}$

Translocas também são mais do que viajantes transnacionais; somos mediadoras culturais, políticas e teóricas. Somos agentes de transculturação. Como contraponto às teorias assimilacionistas da "aculturação", a noção de transculturação de Fernando Ortiz "envolve necessariamente a perda ou o desenraizamento de uma cultura prévia, que pode ser definida como desculturação [... e] traz a ideia da consequente criação de um novo fenômeno cultural" ${ }^{24}$ Lima Costa sugere que, assim como as teorias viajantes e outros fluxos transfronteiriços, o feminismo translocal, pelo menos potencialmente, "desfigura, deforma e transforma a cultura e/ou a disciplina que o recebe". ${ }^{25}$ Translocas interrogam e desestabilizam noções de raça, classe, sexualidades, gêneros e outras "políticas locacionais" em todos os lados das fronteiras, pois essas noções mudam quando nos movemos por essas diversas localidades. Nós desenhamos novas formas raciais e sexuais ao cruzarmos múltiplas fronteiras. ${ }^{26}$ E nossas "remessas" - na maior parte das vezes fielmente enviadas por mulheres migrantes, como bem nota Carrillo $^{27}$ - não são apenas materiais, mas também socioculturais e políticas. ${ }^{28}$ 
29 Anibal QUIJANO, 2000; Walter MIGNOLO, 2000; Enrique DUSSEL, 1994. Para trabalhos recentes de vários autores associados ao grupo de estudos descoloniais, ver a edição especial de Cultural Studies sobre "Coloniality of Power and Decolonial Thinking" (MIGNOLO, 2007).

${ }^{30} \mathrm{KLAHN}$, no prelo.

${ }^{31}$ SOUZA SANTOS, 2002, citado por SCHMIDT, no prelo.

${ }^{32}$ Ver LAÓ-MONTES e BUGGS, no prelo; Victoria BAÑALES, no prelo; Ana Rebeca PRADA, no prelo; e Maylei BLACKWELL, no prelo.

${ }^{33}$ BLACKWELL, no prelo.
Uma conceitualização transloca da transculturação - que promove processos tanto intraculturais quanto transculturais de transformação multidirecional e processos de vários níveis de "desculturação" e re-fundação cultural também objetiva interromper e re-engajar a teorização contemporânea sobre interculturalidad ou interculturalidade..$^{29}$ Como proposto por Norma Klahn, ${ }^{30}$ para melhor entender a "colonialidade do poder" deve-se começar pela compreensão das "traduções e viagens desiguais das práticas, teorias e textos feministas e sua recepção". Citando Boaventura de Souza Santos, Schmidt também insiste que teorias pós-coloniais/descoloniais exigem uma “'densa articulação com a questão da discriminação racial e feminismo', de maneira a revelar as normas sexistas da sexualidade que quase sempre colocam o homem branco e a mulher negra na cama, mas não a mulher branca e o homem negro". ${ }^{31}$ Apesar de uma política translocal de tradução ser crucial para a virada descolonial, a noção do "intercultural" corre o risco de homogeneizar "culturas" subalternas e de ignorar ou desvalorizar diferenças sexuais, de gênero, raça, classe e idade, e relações de poder que sustêm hierarquias mesmo entre sujeitos descoloniais, como indígenas e afro-descendentes. ${ }^{32}$ Maylei Blackwell esclarece, por exemplo, que mulheres indígenas "não apenas se organizam cruzando fronteiras dos Estados-nação, mas também atravessando diferenças sociais, culturais e estruturais internas". ${ }^{33}$

Em suma, defendemos que a análise de viagens e traduções de teorias, práticas e discursos feministas nas Américas pode oferecer perspectivas revigoradas sobre questões tipicamente recortadas em termos do transnacionalismo e novas formas de pensar sobre conexões translocais entre feminismo do norte global e (dentro e através do) sul global. Nosso projeto visa promover uma episteme feminista e antirracista renovada para re-imaginar e reteorizar os Estudos da América Latina travestidos para as Américas globalizadas e transmigrantes do século XXI. Também sinaliza para a possibilidade de transformar os Estudos sobre os Estados Unidos e os Estudos LatinoAmericanos, para que entendam as Américas como uma transfronteira dinâmica, uma formação cultural translocal, ao invés de um espaço geopolítico claramente delineado. Conclamamos ativistas, agentes culturais e produtores de conhecimento, de dentro e de fora da academia, para juntarem-se a nós para traduzir e translocalizar discursos, políticas e práticas subalternos e hegemônicos e para forjar Américas Latinas genuinamente justas e feministas, nos pontos de vista social, sexual, racial, econômico e ambiental. 


\section{Referências bibliográficas}

ASHER, Kiran. "Texts in Context: Afro-Colombian Women's Activism in the Pacific Lowlands of Colombia." In: ALVAREZ, Sonia E., LIMA COSTA, Claudia de, FELIÚ Verónica, HESTER Rebecca, KLAHN Norma, and MILLIE Thayer (eds.), with BUENO, Cruz C. Translocalities/Trans/ocalidades: Feminist Politics of Trans/ation in the Latin/a Américas. Forthcoming.

BAÑALES, Victoria M. “'Women With Guns': Translating Gender in I, Rigoberta Menchú." In: ALVAREZ, Sonia E. et al. (eds.). Translocalities/Translocalidades: Feminist Politics of Trans/ation in the Latin/a Américas. Forthcoming.

BLACKWELL, Maylei. "Translenguas: Mapping the Possibilities and Challenges of Transnational Women's Organizing across Geographies of Difference." In: ALVAREZ, Sonia E. et al. (eds.). Translocalities/Translocalidades: Feminist Politics of Trans/ation in the Latin/a Américas. Forthcoming.

CANDELARIO, Ginetta E. B. Black Behind the Ears: Dominican Racial Identity from Museums to Beauty Shops. Durham, NC: Duke University Press, 2007.

CARRILLO, Teresa. "Feminist Contradictions in the Transnationalization of Domestic Service." In: ALVAREZ, Sonia E. et al. (eds.). Trans/ocalities/Trans/ocalidades: Feminist Politics of Trans/ation in the Latin/a Américas. Forthcoming.

CÉSPEDES, Karina Lissette. "Talking Across Latinidades: Disindentificatory Feminism in El Mundo Surdo." In: MIRABAL, Nancy R., and LAÓ-MONTES, Agustín (eds.). Technofuturos: Critical Interventions in Latina/o Studies. New York: Lexington Books, 2007.

DAVIS, Kathy. The Making of Our Bodies, Our Selves: How Feminism Travels across Borders. Durham: Duke University Press, 2007.

DUANY, Jorge. "The Puerto Rican Diaspora: Transnational Circuits of Culture, Politics, and Economics." In: The Hospitable U.S., University of Connecticut, Storrs, 2008. Proceedings.

DUSSEL, Enrique. "1492: El Encubrimiento del Otro: Hacia el Origen del 'Mito de la Modernidad'." Latino América, n. 25, p. 7-121, 1994.

ESPINAL, Isabel. "El Incansable Juego/The Untiring Game: Dominican Women Writing and Translating Ourselves." In: ALVAREZ, Sonia E. et al. (eds.). Trans/ocalities/Trans/ocalidades: Feminist Politics of Translation in the Latin/a Américas. Forthcoming.

GREWAL, Inderpal, and KAPLAN, Caren. "Introduction: Transnational Feminist Practices and Questions of Postmodernity." In:___ (eds.). Scattered Hegemonies: Postmodernity and Transnational Feminist Practices. Minneapolis: University of Minnesota Press, 1994. 
HARCOURT, Wendy, and ESCOBAR, Arturo (eds.). Women and the Politics of Place. Bloomfield, CT: Kumarian Press, Inc., 2005.

KLAHN, Norma. "Locating Women's Writing in the Age of Latinamericanismo and Globalization." In: ALVAREZ, Sonia E. et al. (eds.). Trans/ocalities/Trans/ocalidades: Feminist Politics of Trans/ation in the Latin/a Américas. Forthcoming.

LAÓ-MONTES, Agustín. "Afro-Latinidades: Bridging Blackness and Latinidad." In: MIRABAL, Nancy R., and LAÓ-MONTES, Agustín (eds.). Technofuturos: Critical Interventions in Latina/o Studies. New York: Lexington Books, 2007.

LAÓ-MONTES, Agustín, and BUGGS, Mirangela. "Translocal Space of Afro-Latinidad: Critical Feminist Visions for Diasporic Bridge-Building." In: ALVAREZ, Sonia E. et al. (eds.). Trans/ocalities/Trans/ocalidades: Feminist Politics of Trans/ation in the Latin/a Américas. Forthcoming.

LIMA COSTA, Claudia de. "Being Here and Writing There: On the Limits of Theory and Lived Experience." Signs: Journal of Women in Culture and Society, v. 25, n. 3, 2000. p. 728759.

"Lost (and Found?) in Translation: Feminisms in Hemispheric Dialogue." In: ALVAREZ, Sonia E. et al. (eds.). Trans/ocalities/Translocalidades: Feminist Politics of Trans/ation in the Latin/a Américas. Forthcoming.

LUGONES, Maria. "Playfulness, 'World' Traveling, and Loving Perception." In: ANZALDÚA, G. (ed.). Making Face, Making Soul/Haciendo Caras: Creative and Critical Perspectives by Women of Color. San Francisco: Aunt Lute Press, 1990.

MAIA, Suzana. "Performing Seduction and National Identity: Brazilian Erotic Dancers in New York." In: ALVAREZ, Sonia E. et al. (eds.). Translocalities/Trans/ocalidades: Feminist Politics of Trans/ation in the Latin/a Américas. Forthcoming.

MIGNOLO, Walter. Local Histories/Global Designs: Coloniality, Subaltern Knowledges, and Border Thinking. Princeton, NJ: Princeton University Press, 2000.

MIGNOLO, Walter D. Coloniality of Power and De-colonial Thinking. Cultural Studies, v. 21, n. 2-3, 2007. Introduction.

MILLÁN, Márgara. "Politics of Translation in Mexican Contemporary Feminism." In: ALVAREZ, Sonia E. et al. (eds.). Translocalities/Trans/ocalidades: Feminist Politics of Trans/ation in the Latin/a Américas. Forthcoming.

PRADA, Ana Rebeca. "Is Anzaldúa Translatable in Bolivia?" In: ALVAREZ, Sonia E. et al. (Eds.). Trans/ocalities/Trans/ocalidades: Feminist Politics of Translation in the Latin/a Américas. Forthcoming.

QUIJANO, Anibal. "Coloniality of Power, Eurocentrism, and Latin America." Nepantla, v. 1, n. 3, 2000. p. 139-155.

RENTA, Priscila. "Salsa Dance Performance: Latina/o History in Motion." In: MIRABAL, Nancy R., and LAÓ-MONTES, Agustín 
(eds.). Technofuturos: Critical Interventions in Latina/o Studies. New York: Lexington Books, 2007.

SANDOVAL, Chela. "U.S. Third World Feminism: The Theory and Method of Oppositional Consciousness in the Postmodern World." Genders, n. 10, Spring 1991. p. 1-24.

Methodology of the Oppressed. Minneapolis: University of Minnesota Press, 2000.

SCHMIDT, Simone. "Cravo Canela, Bala e Favela." In: ALVAREZ, Sonia E. et al. (eds.). Trans/ocalities/Trans/ocalidades: Feminist Politics of Translation in the Latin/a Américas. Forthcoming.

SHAPIRO, Ester. "'Your Spanish is too Caribbean, Strange to the Sensibilities of Western Thought:' A U.S. Latina Lens on Hope and Heartbreak In Translating Our Bodies, Ourselves as a Transnational Feminist Text." In: ALVAREZ, Sonia E. et al. (eds.). Translocalities/Translocalidades: Feminist Politics of Trans/ation in the Latin/a Américas. Forthcoming.

THAYER, Millie. "Translating against the Market: Transposing and Resisting Meanings as Feminist Political Practice." In: ALVAREZ, Sonia E. et al. (eds.). Trans/ocalities/Trans/ocalidades: Feminist Politics of Translation in the Latin/a Américas. Forthcoming.

[Recebido em outubro de 2009 e aceito para publicação em outubro de 2009]

\section{Enacting a Translocal Feminist Politics of Translation}

Abstract: Our collective project on Translocalities/Translocalidades: Feminist Politics of Translation in the Latin/a Américas explores how feminist discourses and practices travel across a variety of sites and directionalities to become interpretive paradigms to read/write issues of class, gender, race, sexuality, migration, health, social movements, citizenship, politics, and the circulation of identities and texts. Translation is politically and theoretically indispensable to forging feminist, anti-racist, postcolonial/postoccidentalist political alliances and epistemologies because the Latin/a Américas - as a transborder cultural formation rather than a territorially delimited one must be understood as translocal in a dual sense. The first sense we will deploy - that of translocation - builds on but moves beyond US Third World feminist conceptions of the "politics of location." Rather than "immigrating" and "assimilating," moreover, many people in the Latin/a Américas increasingly move back and forth between localities, between historically situated and culturally specific, though increasingly porous, places, across multiple borders, and not just between nations (as implied in the term "transnational migration," for instance). We therefore deploy the notion of translocal in a second sense, which we call translocalities/translocalidades, precisely to capture these multidirectional crossings and movements.

Key Words: Trans/ation; Transnational Feminisms; Race/Racism; Latin American Feminisms; Latinas. 